\title{
Broadband Plasmonic Couplers for Light Trapping and Waveguiding
}

\author{
F. Djidjeli*a ${ }^{\mathrm{a}}$, E. Jaberansary ${ }^{\mathrm{a}}$, H. M. H. Chong ${ }^{\mathrm{a}}$, and D. M. Bagnall ${ }^{\mathrm{a}}$ \\ ${ }^{a}$ Nano Research Group, School of Electronics and Computer Science, University of \\ Southampton, Highfield Campus, Southampton SO17 1BJ, United Kingdom.
}

\begin{abstract}
We have experimentally and theoretically demonstrated plasmonic couplers based on metal nanoparticles film (metal islands) deposited upon the optical dielectric waveguide. These nanoparticles forward scatter radiation into the optical waveguide that would otherwise be reflected and transmitted. Design, fabrication and optimisation of the plasmonic silver islands and tantalum pentoxide waveguide are reported. Broadband characteristics of light transmission showing plasmons excitation have been measured and in good agreement with simulated results from $400 \mathrm{~nm}$ to $800 \mathrm{~nm}$. Coupling spectrum up to $15 \%$ is measured for the plasmonic coupler and in good agreement with the simulated results.
\end{abstract}

Keywords: Plasmon, coupler, waveguide, nanoparticle

\section{INTRODUCTION}

When noble metal nanoparticles are excited by an electromagnetic wave (visible and IR light), they exhibit collective oscillations of their conduction electrons, known as localized surface plasmons. The spectral position of the plasmons resonance depends on the nanoparticle geometry, particles distribution, metal composition and the surrounding environment. Hence, altering these properties can tune the surface plasmons resonance to a desired wavelength band. The nanoparticle geometrical shape can have a spherical, triangular, rectangular and even tetrahedron shape; these shapes can scatter the incident light into specific frequency. Additionally, a spectrum redshift was seen with the increasing particle size [1]. Typical metals that support surface plasmons are silver (Ag), gold $\mathrm{Au}$ ), copper, titanium, chromium and aluminum. Ag and Au are most commonly used metals in current research, due to their characteristics and spectral responses at visible and NIR spectrum [1]. Various techniques of nanoparticles fabrication such as metal-island films, e-beam lithography, and chemical synthesis are used by many research groups [2-4]. Each technology has its advantages and disadvantages with respect to the nanoparticles quality and the fabrication cost.

In optoelectronic, the most widely used guiding mechanism is the total internal reflection; and planar waveguides are their basic elements. Several materials are used for waveguiding, among them: silicon nitride and tantalum pentoxide. These transparent dielectric materials are well-known for very broadband light guiding applications. For fabrication process, there are different methods for waveguide core-film deposition, as a good example RF magnetron sputtering method. Tantalum pentoxide $\left(\mathrm{Ta}_{2} \mathrm{O}_{5}\right)$ was originally used in anti-reflection coatings for solar cells, and later it was introduced in waveguiding application. Due to its characteristics: low absorption, high refractive index, chemical and thermal stability [5]; this material is now widely used as optical waveguide [6].

Plasmonic nanoparticles has been used to couple propagating plane waves of solar radiation into propagating modes of a

semiconductor and SOI [7] and potentially increase the absorption of light in solar cells [8]. In this paper, we are going to combine the two systems, where the spectral response of the combination is analyzed and studied experimentally and theoretically. A fabricated plasmonic coupler is analyzed experimentally using an integrating sphere [9], and modeled using 3D FDTD software from Lumerical [10]. A comparison is then presented showing a good agreement of the experimental and theoretical results. 
*fd07r@ecs.soton.ac.uk; phone 00442380593737.

\section{PLASMONIC COUPLER MODEL}

In this work, plasmonic coupler was achieved by depositing metal-island films onto an optical dielectric waveguide. The complete structure is shown in Figure 1, where the plasmonic islands are deposited upon planar dielectric waveguide. Interaction of the metal islands with the incident electromagnetic radiation would induce the scattered light which will be then trapped and confined in the waveguide by total internal reflection. Thus, the coupling and the guiding mechanisms of the electromagnetic radiation are accomplished.

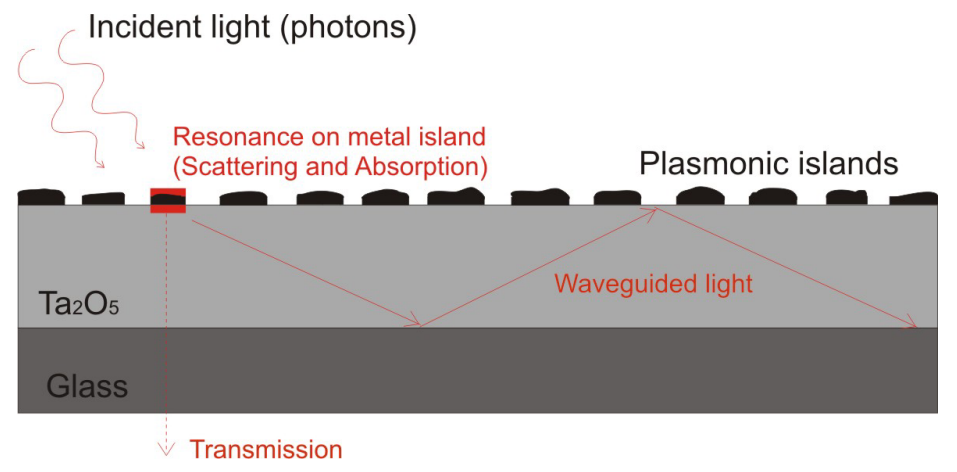

Figure 1. A schematic representation of the plasmonic islands/waveguide coupling configuration.

$\mathrm{Ag}$ is the metal chosen for this work, due to its characteristics, high scattering and comparatively low absorption compared to other metals such as Au. Metal film islanding technique is used for the formation of the Ag plasmonic layer. This technique has the simplest and cheapest process compared to the cited techniques.

To understand better the nature of interaction with the incident light, we have theoretically and experimentally compared the scattering responses for the $\mathrm{Ag}$ and $\mathrm{Au}$ nanoparticles; and found that the $\mathrm{Ag}$ metal interacts strongly with light by means of scattering phenomenon compared to Au metal. Figure 2(a) illustrates the comparison between the scattering efficiencies for $\mathrm{Ag}$ and $\mathrm{Au}$ nanospheres of $30 \mathrm{~nm}$ radius in glass medium (using MiePlot software [11]). The spectral position of the surface plasmons resonance peak is at the wavelength of $452 \mathrm{~nm}$ and $567 \mathrm{~nm}$ for the $\mathrm{Ag}$ and $\mathrm{Au}$, respectively. Figure 2(b) shows the measured scattering spectra for 10nm Ag and Au uniform layer deposited on glass substrate and annealed at the temperature of $300^{\circ} \mathrm{C}$ for 1 hour period time. For these metal islands, the spectral position of the surface plasmons resonance peak is at the wavelength of $496 \mathrm{~nm}$ and $635 \mathrm{~nm}$ for the $\mathrm{Ag}$ and $\mathrm{Au}$, respectively.
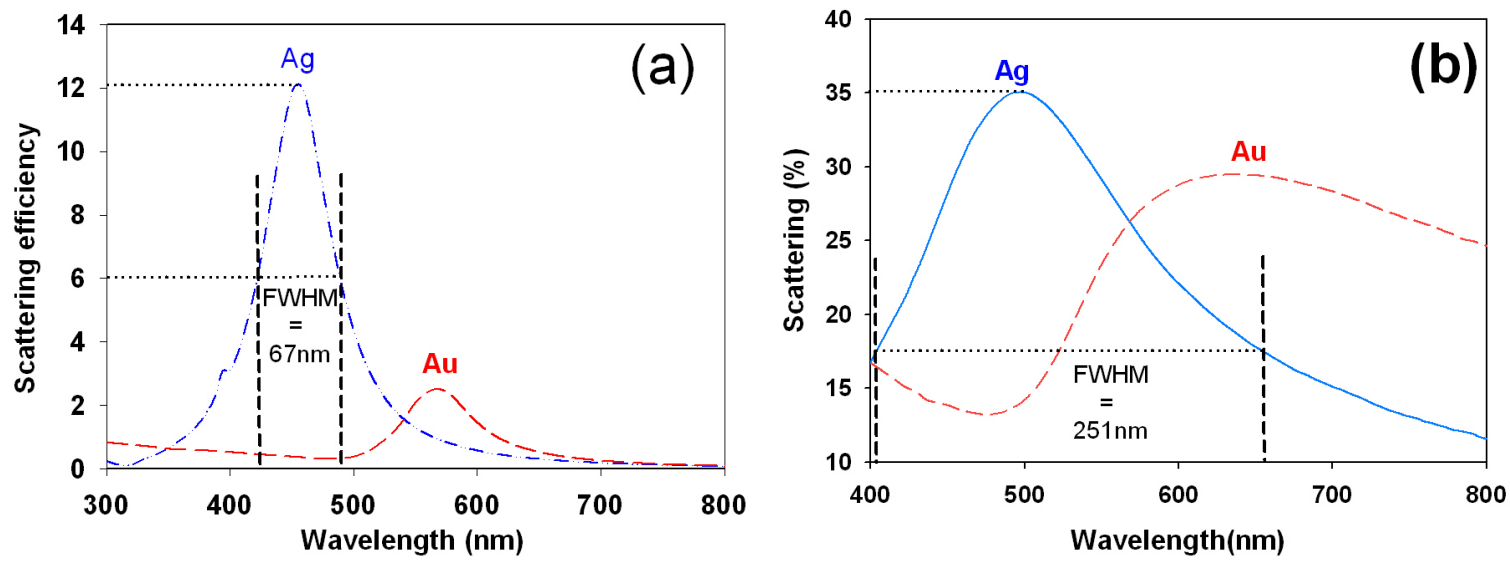

Figure 2: Comparison of scattering spectra of Ag and Au nanoparticles (a) simulation (b) measurement. 
For the plasmonic coupler we used Ag metal and experimentally showed that it scatters more the incident light than the Au metal (Figure 2(b)). An optimum forming process of the Ag islands was achieved after analysing different samples with different Ag uniform layer thicknesses, temperatures and annealing periods grown on glass substrate. An impressive extinction of the beam incident on the plasmonic layer has been achieved with $10 \mathrm{~nm} \mathrm{Ag} \mathrm{uniform}$ layer, $350^{\circ} \mathrm{C}$ annealing temperature and 1 hour annealing period characteristics. Figure 3 illustrates the absorption, extinction and scattering spectra of this chosen plasmonic layer, where up to $35 \%$ of the incident light is scattered.

Full-width half-maxima (FWHM) were found to be narrower for Ag compared to Au. From Figure 2, wider FWHW is seen for both metal islands compared to the spherical geometry. Thus using the metal islanding technique induces broadband optical responses. Additionally, the spectral position of the resonance peak is seen to red-shift for the Ag islands.

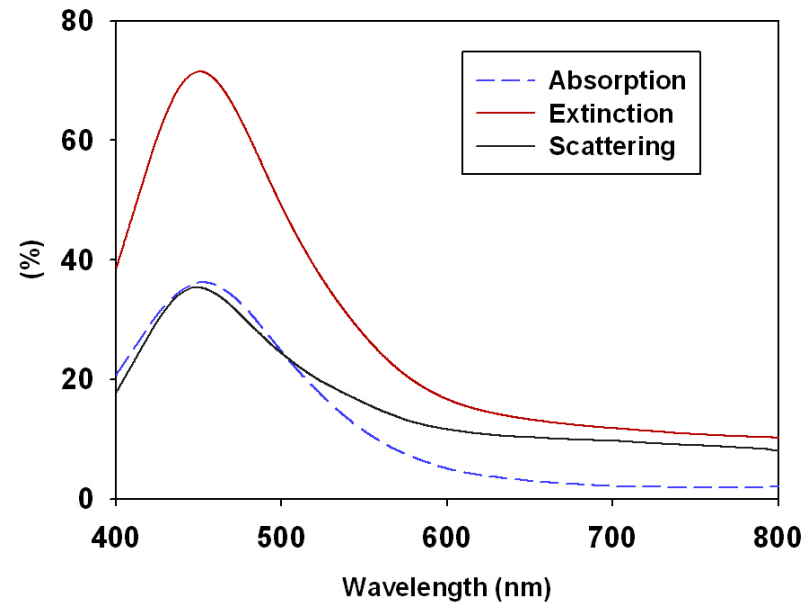

Figure 3: Absorption, extinction and scattering spectra of plasmonic layer.

Tantalum pentoxide $\left(\mathrm{Ta}_{2} \mathrm{O}_{5}\right)$ is used for the waveguiding core because of its low absorption and high refractive index characteristics. The complete plasmonic coupler would be the Ag plasmonic layer deposited upon the $\mathrm{Ta}_{2} \mathrm{O}_{5}$ waveguide. Starting from Maxwell's equations, a theoretical analysis using the effective index method is performed for different waveguide core thicknesses. The different modes supported by the waveguides were calculated.

Prism coupler was used to measure and visualise the different modes supported by these waveguides. Using this method, a refractive index of 2.1 was measured at $633 \mathrm{~nm}$ for the $\mathrm{Ta}_{2} \mathrm{O}_{5}$ waveguide. Comparing the theoretical and experimental analysis, the $300 \mathrm{~nm}$ thick waveguide found to be the best. Thus for the plasmonic coupler, the $300 \mathrm{~nm}$ core thickness waveguide is used.

\section{Fabrication process}

Metal-island films approaches are potentially economical and large scale fabrication techniques compared to other methods such as e-beam lithography. Plasmonic islands are formed by depositing a thin uniform layer of metal, using thermal evaporation technique, followed by annealing in nitrogen for specific temperature and period of time. Using thermal evaporation technique, the deposition rate for the Ag uniform layer was adjusted to around $0.01 \mathrm{~nm} / \mathrm{s}$, and thus achieving a good formation of the $\mathrm{Ag}$ islands. The annealing process was carried out in a tube furnace.

In this paper, $\mathrm{RF}$ magnetron sputtering was employed to deposit $\mathrm{Ta}_{2} \mathrm{O}_{5}$ thin films on glass substrates, at the base pressure of the order of $10^{-7}$ torr, magnetron power of $300 \mathrm{~W}$ and $\mathrm{O}_{2}$ and Ar flow rates of 5 and $20 \mathrm{sccm}$, respectively. Optimization of the substrate temperature was carried out, and the layer sputtered at the substrate temperature of $200^{\circ} \mathrm{C}$ has the maximum propagation length. From the waveguide theoretical analysis, the $\mathrm{Ta}_{2} \mathrm{O}_{5}$ core thickness of $300 \mathrm{~nm}$ was chosen for this work.

The $10 \mathrm{~nm}$ Ag uniform layer is then evaporated on top of the $300 \mathrm{~nm} \mathrm{Ta}_{2} \mathrm{O}_{5}$ waveguide and the whole device was annealed at $350^{\circ} \mathrm{C}$ for 1 hour and the plasmonic coupler is formed. 


\subsection{SEM image analysis and processing}

The nanostructural changes that take place in the Ag thin-film after annealing process can be shown by SEM images. Figure 4 shows the SEM image of the top view of the Ag plasmonic islands of $10 \mathrm{~nm}$ layer on top of $\mathrm{Ta}_{2} \mathrm{O}_{5}$ waveguide grown on glass substrate and its image analysis.
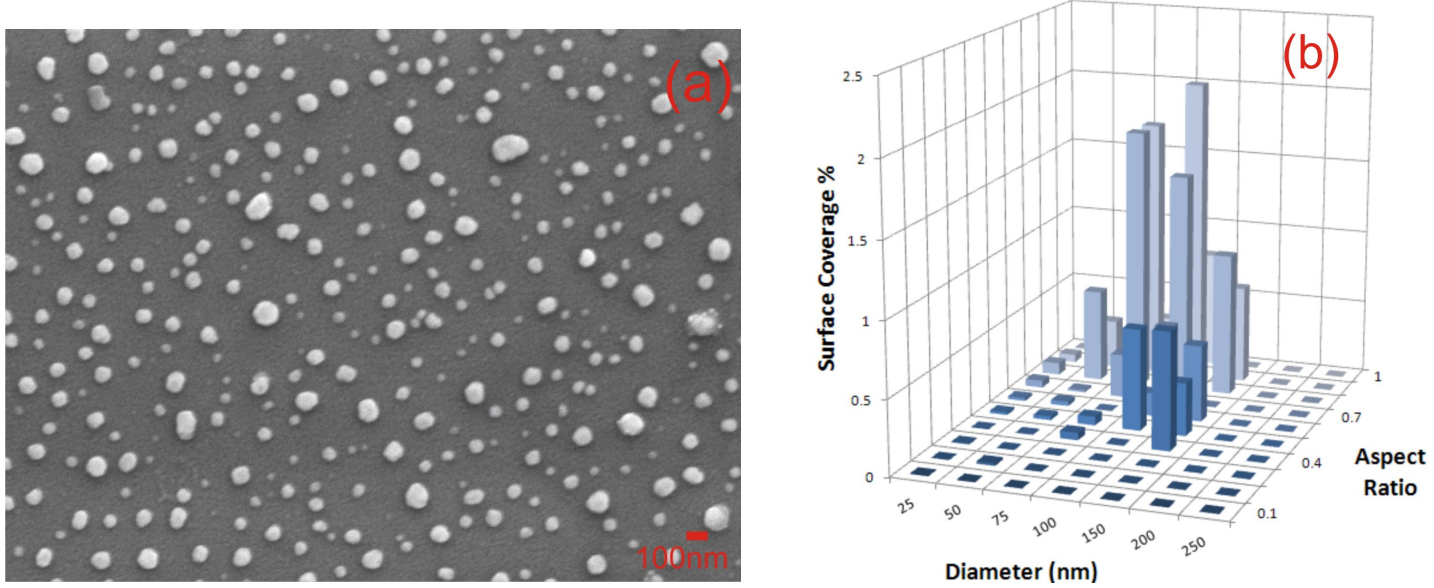

Figure 4: SEM image and 3D histogram analysis of Ag islands (on top of 300nm thick $\mathrm{Ta}_{2} \mathrm{O}_{5}$ ).

Image analysis was performed on the islands SEM image using ImageJ software [12]. Surface coverage, area, circularity, diameter and aspect ratio parameters are calculated and obtained by ImageJ. Figure 4(b) shows the Ag islands equivalent diameter which is in the distribution range of $75-150 \mathrm{~nm}$, giving an average of $100 \mathrm{~nm}$ diameter. From this figure, the aspect ratio of the $\mathrm{Ag}$ islands is mostly around $0.8-0.9$; thus, the $\mathrm{Ag}$ islands tend to have a rounded particle shape.

At all sizes investigated in this study, it is apparent that larger nanoparticles scatter light much strongly than small nanoparticles. These range of $\mathrm{Ag}$ islands diameters are good sizes (larger enough) for the scattering of the incident beam of light.

For better analysis and interpretation, we have done a comparison study of two different Ag film thicknesses of $7.5 \mathrm{~nm}$ and $20 \mathrm{~nm}$. The annealed Ag islands for both thicknesses are shown in Figure 5. Figure 6 illustrates their corresponding image analysis.
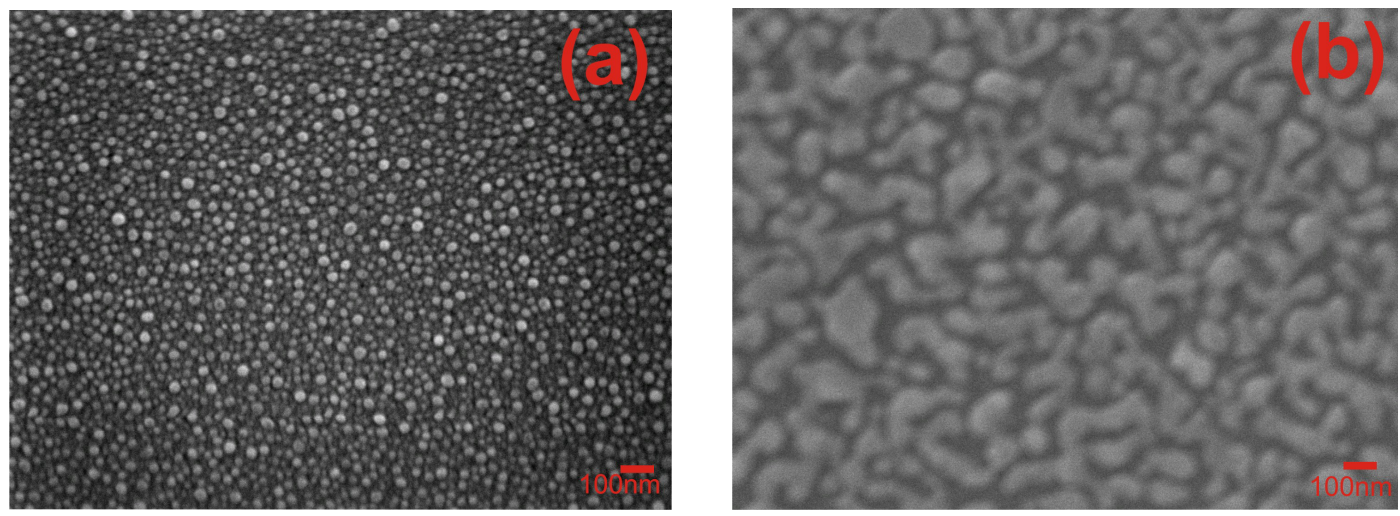

Figure 5: SEM image of Ag islands of uniform thickness (a) $7.5 \mathrm{~nm}$ and (b) $20 \mathrm{~nm}$.

For the $7.5 \mathrm{~nm}$ sample (Figure 5(a)), the Ag islands are dense and have very small diameter. Figure 6(a) confirms and shows the high density of the islands in the range of $25 \mathrm{~nm}$ to $75 \mathrm{~nm}$ with an average diameter of $50 \mathrm{~nm}$. Additionally, the $\mathrm{Ag}$ islands have a high percentage at the aspect ratio of 0.9 . Thus, the islands have mostly a 
circular shape .For the second sample (20nm Ag thickness), it is seen from Figure 5(b), a poorly formal regular island. Thus, coalescence of Ag islands has poorly taken place. Figure 6(b) demonstrates that the islands density is very poor and their average diameter is around $175 \mathrm{~nm}$. Moreover, the aspect ratio is in the range of 0.2 to 0.7 which means a non-spherical shape.
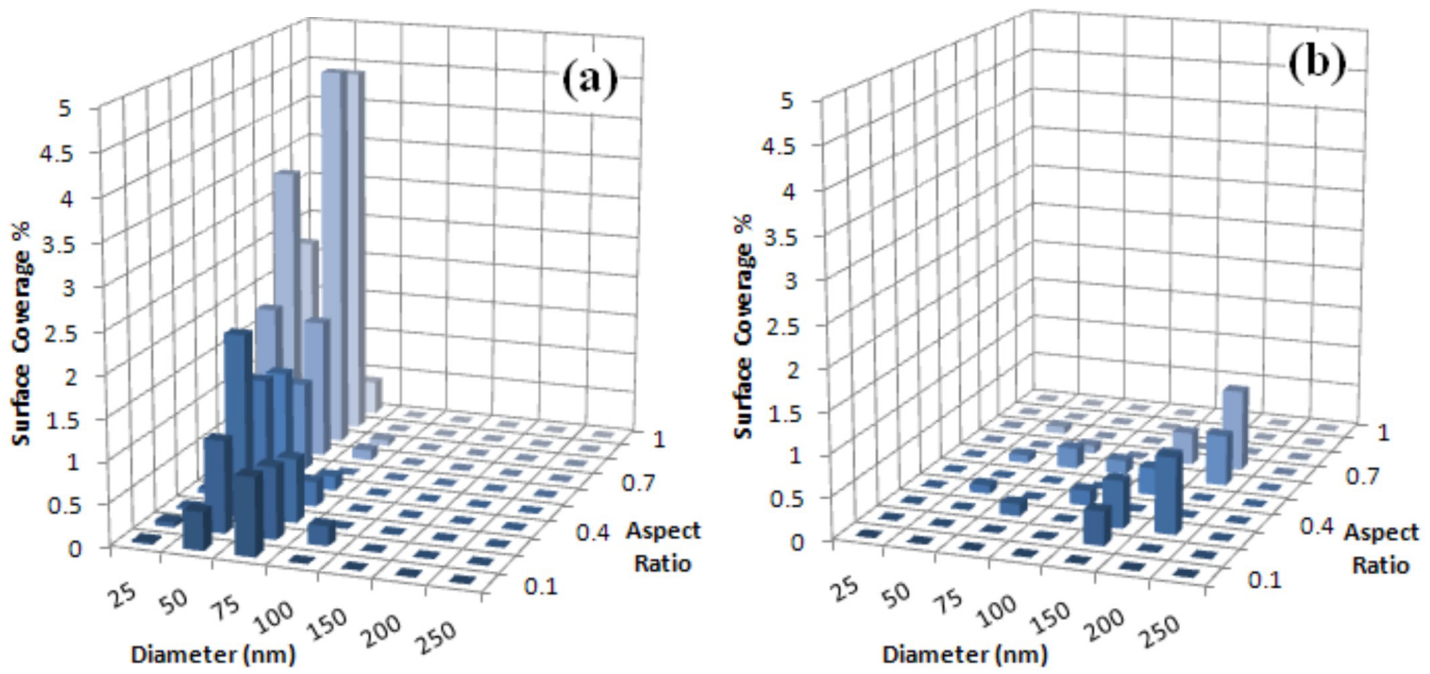

Figure 6: 3D histogram for the diameter and aspect ratio of the Ag islands of (a) $7.5 \mathrm{~nm}$ and (b) 20nm uniform layer thickness.

It can be concluded from this analysis that the $10 \mathrm{~nm}$ Ag layer has the best islands formation in term of light scattering characteristic.

\section{FDTD modeling of plasmonic coupler}

The plasmonic coupler structure was simulated using commercial 3D FDTD software from Lumerical. In this work, the 2D SEM image of the fabricated Ag islands in Figure 4 was first converted to 3D FDTD model using Matlab. Due to the size of the nanoparticles, the grayscale SEM 2D image suffered from low colors contrast, especially at the boundaries of the semi spherical particles. Therefore, the 2D image was rendered to 3D image in Matlab based on the algorithm that adjust the contrast and brightness threshold level of the image and isolate colors below the threshold value. The Ag islands of the SEM were then mapped out with corresponding coordinates and assigned with information such as length, position, thickness and width. Finally the 3D metal islands are reshaped as spheres, oblate or prolate spheroid according to the assigned dimensions. The result of the plasmonic coupler of Figure 4 SEM 3D conversion with an area of $3.4 \mu \mathrm{m} \times 2.6 \mu \mathrm{m}$ for FDTD simulation is shown in Figure 7.

A plane wave source with wavelength from $400 \mathrm{~nm}$ to $1000 \mathrm{~nm}$ is used to study the transmission characteristic and scattering effects of the plasmonic coupler into the $\mathrm{Ta}_{2} \mathrm{O}_{5}$ waveguide. The source is placed on top of the plasmonic coupler to simulate a visible light source being launched into the X-Y plane of the structure.
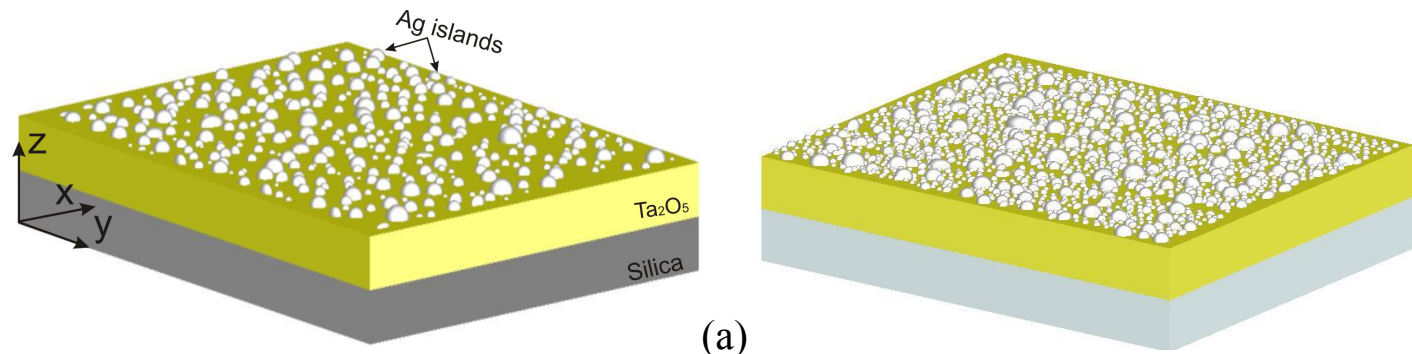

(a)

Figure 7: 3D model of the simulated plasmonic coupler of fabricated (a) 10nm and (b) $7.5 \mathrm{~nm}$ thick uniform Ag layers. 


\section{RESULTS \& DISCUSSION}

\section{Measurements results}

The plasmonic coupler was analyzed by means of spectrometry technique. The light from a tungsten-halogen lamp was directed and focused onto the Ag islands, where the sample was held in three positions of the integrating sphere (Figure 8). The signals detected from the different positions are collected by a spectrometer connected to a computer.
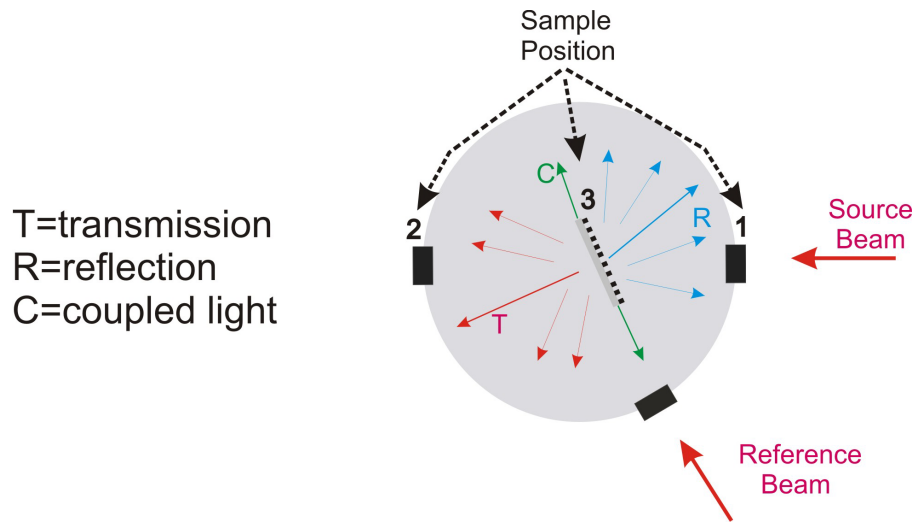

Figure 8. Schematic for the integrating sphere (in double beam mode).

Figure 8 illustrates positions 1 and 2 of the integrating sphere to measure the transmitted and reflected light, respectively. In position 3, the plasmonic coupler specimen is placed in the centre of the sphere, and the transmitted, reflected and coupled light are characterized simultaneously. In this position (3), the sample is tilted a few degrees away from the normal incident angle to avoid reflection loss from the Ag islands (see, Figure 8). The coupled light spectrum is first obtained by subtracting the transmitted and reflected lights signals, which is empirically obtained and processed by the integrated sphere measurement system (with the reference beam included).

The measured results: transmitted, reflected and coupled light are shown in Figure 9(a). From this figure, the Ag islands exhibit high optical coupling up to $15 \%$ into the dielectric waveguide at $545 \mathrm{~nm}$.
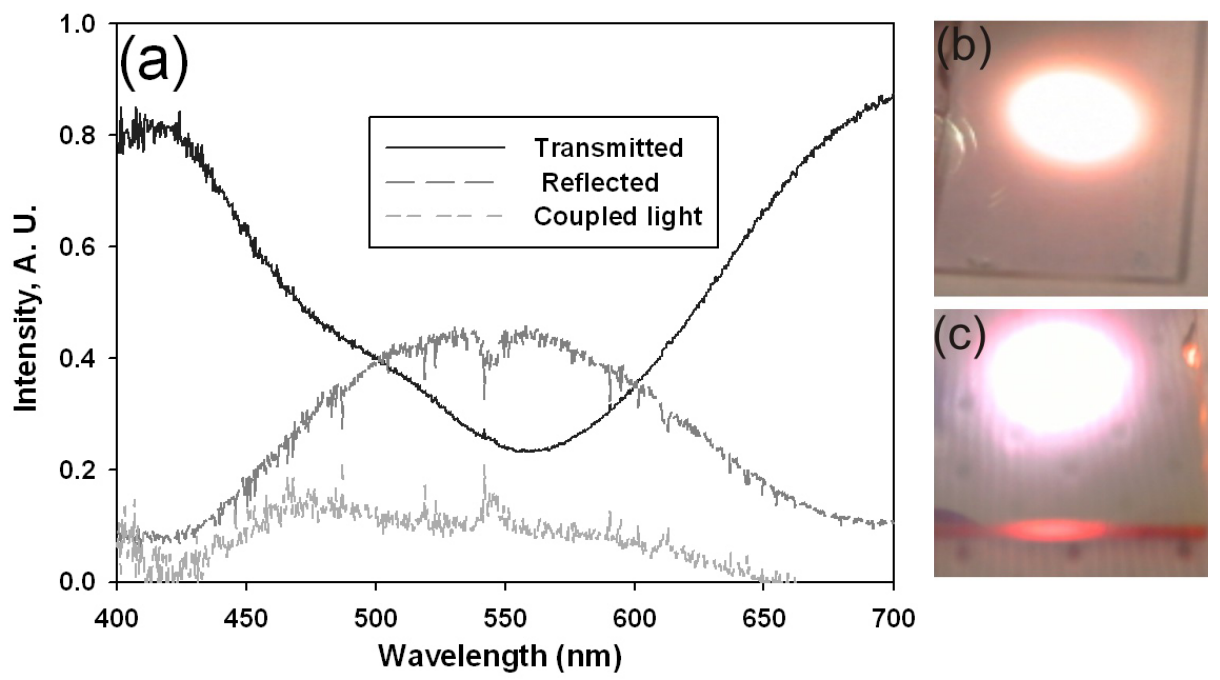

Figure 9: Pictures showing (a) measured transmittance, absorptance, reflectance, and coupled light of the islands grown onto the waveguide; white light incident on waveguides sample (b) without plasmonic islands and (c) with Ag islands. 


\subsection{Analysis of the Optical and simulation results}

Figure 9(a) shows the dip in the transmission at $555 \mathrm{~nm}$, with a bandwidth of $178 \mathrm{~nm}$. Whereas, the measured reflected light illustrates a peak at $558 \mathrm{~nm}$ and a bandwidth of $179 \mathrm{~nm}$. The system has a coupling broadband ranging from $350 \mathrm{~nm}$ to $650 \mathrm{~nm}$ wavelengths, with a bandwidth of $150 \mathrm{~nm}$, which corresponds to the coupled intensity signal. A broadband optical response with high optical coupling in the visible region is seen from the measured plasmonic coupler. An optical system with large coupling, transmission, and reflection broadbands in the visible region would be of high interest for the waveguiding applications.

Figures 9(b) and 9(c) illustrate a clear optical demonstration of the light coupling phenomenon. When a white light source is incident on the waveguide without plasmonic islands, we see no evidence of coupling light at the edge of the specimen (Figure 9(b)). However, at the presence of the Ag islands, predominantly red light is seen to emerge from the side of the sample (Figure 9(c)). Using the FDTD simulation, comparison is carried out between Ag islands transmission for the uniform layer thicknesses of $7.5 \mathrm{~nm}$ and $10 \mathrm{~nm}$ (Figure 10(a)). The corresponding SEM images for the Ag islands are shown in Figure 4(a) and Figure 5(a).

Comparing both transmission curves, we see the same trend for the two simulated plasmonic couplers. However, the bandwidth of the $10 \mathrm{~nm}$ thick Ag layer is wider. Additionally, the $7.5 \mathrm{~nm}$ layer has deeper resonance, which is due to the increase in the absorption of incident light for the dense $\mathrm{Ag}$ islands and reducing the light scattering into the $\mathrm{Ta}_{2} \mathrm{O}_{5}$ waveguide. This increase in absorption phenomenon occurs because of the small size of the Ag islands. It is again demonstrated that smaller size metal islands absorb more light than the larger ones. From this analysis, the $10 \mathrm{~nm} \mathrm{Ag}$ thick layer is the best thickness for this plasmonic coupler

Finally, Figure 10(b) demonstrates that the agreement between numerical and experimental results is excellent for the plasmonic coupler (transmission characteristic).
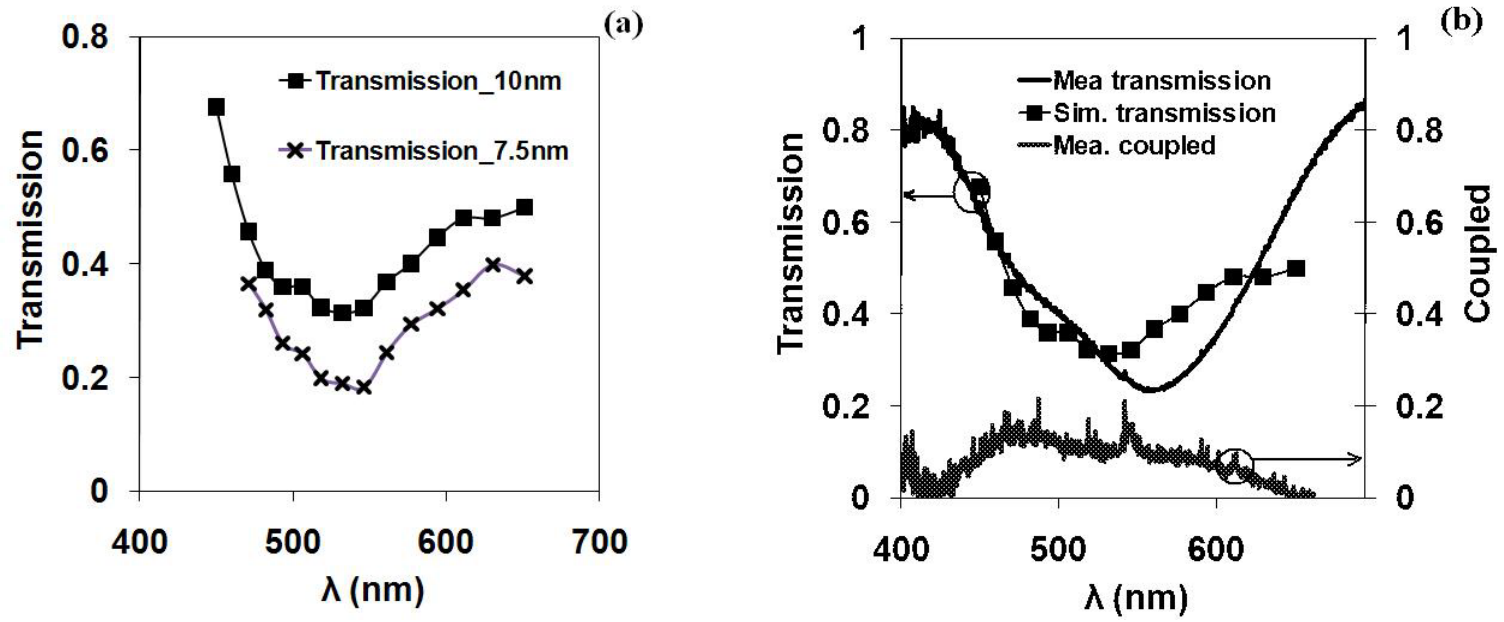

Figure 10: Transmission comparison for (a) the simulation of two different Ag thick layers and (b) Measured and simulated transmission and coupled light for 10nm thick layer.

\section{CONCLUSION}

Metal islands can be surprisingly efficient at coupling light into planar optical waveguide; as has been demonstrated in this work. The radiating Ag islands transfer a significant portion of their energy into the optical waveguide. We have shown experimentally and theoretically the plasmon coupling of the electromagnetic waves into planar dielectric waveguide. The broad resonance in the transmission indicates enhanced radiation transfer by the metal nanoparticles. The broadband coupling could be useful for multiple applications in the visible wavelengths region. The potential applications are high efficiency solar cell and coupler for submicron waveguide structures.

This work is supported by PV SuperGen - PV materials for the $21^{\text {st }}$ century and ISIRAN. 


\section{REFERENCES}

[1] J. J. Mock, M. Barbic, D. R. Smith, D. A. Schultz, and S. Schultz, "Shape effects in plasmon resonance of individual colloidal silver nanoparticles," Journal of Chemical Physics, vol. 116, pp. 6755-6759, Apr 152002.

[2] G. Kalyuzhny, A. Vaskevich, M. A. Schneeweiss, and I. Rubinstein, "Transmission surface-plasmon resonance (TSPR) measurements for monitoring adsorption on ultrathin gold island films," Chemistry-a European Journal, vol. 8, pp. 3850-3857, Sep 22002.

[3] W. Rechberger, A. Hohenau, A. Leitner, J. R. Krenn, B. Lamprecht, and F. R. Aussenegg, "Optical properties of two interacting gold nanoparticles," Optics Communications, vol. 220, pp. 137-141, May 12003.

[4] K. C. Grabar, P. C. Smith, M. D. Musick, J. A. Davis, D. G. Walter, M. A. Jackson, A. P. Guthrie, and M. J. Natan, "Kinetic control of interparticle spacing in Au colloid-based surfaces: Rational nanometer-scale architecture," Journal of the American Chemical Society, vol. 118, pp. 1148-1153, Feb 71996.

[5] K. Schmitt, K. Oehse, G. Sulz, and C. Hoffmann, "Evanescent field sensors based on tantalum pentoxide waveguides A review," Sensors, vol. 8, pp. 711-738, Feb 2008.

[6] B. Unal, M. C. Netti, M. A. Hassan, P. J. Ayliffe, M. D. B. Charlton, F. Lahoz, N. M. B. Perney, D. P. Shepherd, C. Y. Tai, J. S. Wilkinson, and G. J. Parker, "Neodymium-doped tantalum pentoxide waveguide lasers," IEEE Journal of Quantum Electronics, vol. 41, pp. 1565-1573, Dec 2005.

[7] H. R. Stuart and D. G. Hall, "Absorption enhancement in silicon-on-insulator waveguides using metal island films," Applied Physics Letters, vol. 69, pp. 2327-2329, Oct 141996.

[8] K. R. Catchpole and A. Polman, "Plasmonic solar cells," Optics Express, vol. 16, pp. 21793-21800, Dec 222008.

[9] D. D. Evanoff and G. Chumanov, "Size-controlled synthesis of nanoparticles. 2. Measurement of extinction, scattering, and absorption cross sections," Journal of Physical Chemistry B, vol. 108, pp. 13957-13962, Sep 162004.

[10] FDTD Solutions, "http://www.lumerical.com/,"2010.

[11] P. Laven, "MiePlot," www.philiplaven.com/mieplot.htm, 2010.

[12] ImageJ, "http://rsbweb.nih.gov/ij/,"2010. 